Revista Eletrônica do Mestrado

Profissional em Administração da UnP

\title{
edUnp
}

V. 11, N. 2, 2019

ISSN 1984-4204

https://repositorio.unp.br/index.php/raunp

https://doi.org/10.21714/raunp.v11i2.2073

\section{Validando as relações de causa e efeito no balanced scorecard (BSC): um estudo de caso no setor hoteleiro}

\section{Validating cause and effect relationships in balanced scorecard (BSC): a case study in the hotel sector}

\author{
Alessandro Alves Galdino ${ }^{\mathrm{a}}$, Fernanda Stephanie Camurça Chaves ${ }^{\mathrm{b}}$, Alan Santos de Oliveira ${ }^{\mathrm{c}}$ \\ ${ }^{a}$ Graduando em Ciências Contábeis. Universidade Federal do Ceará (UFC). alessandro.agaldino2018@gmail.com

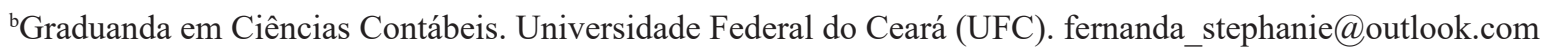

`Mestre em Ciências Contábeis. Professor Assistente da Universidade Federal do Ceará (UFC). asoalansantos@gmail.com

\begin{abstract}
Resumo
O objetivo deste artigo foi verificar a existência da relação de causa e efeito entre os indicadores de desempenho pertencentes ao Balanced Scorecard (BSC) aplicados em um hotel. Utilizou-se uma abordagem metodológica descritiva, bibliográfica, documental e quantitativa, por meio de um estudo de caso. Estatísticas descritivas e análises de modelos lineares foram realizados em 14 indicadores do período de três anos de um hotel localizado em João Pessoa - Paraíba (PB), organizados com base nas perspectivas do BSC: Financeira, Clientes, Processos Internos e Aprendizado e Crescimento. Percebeu-se que indicadores das Perspectivas Clientes e Aprendizagem e Crescimento influenciaram a perspectiva Financeira. Indicadores da perspectiva aprendizagem e crescimento influenciaram os Processos Internos; alguns indicadores dos Processos Internos influenciaram a perspectiva Clientes, bem como alguns indicadores da perspectiva Clientes apresentaram relações com a perspectiva Financeira, revelando indícios de um efeito em cascata. Concluiu-se que existem relações de causa e efeito entre os indicadores estudados, porém as evidências não foram generalizadas entre todos os indicadores. Assim, o estudo contribuiu academicamente com a validação das relações de causa e efeito nas perspectivas do BSC para setor hoteleiro, bem como na prática dos gestores que podem ter auxílio para estruturação do BSC e criação de mapas estratégicos.
\end{abstract}

Palavras-chave: Balanced Scorecard; Causa e efeito; Setor hoteleiro.

\begin{abstract}
The objective of this paper was to verify the existence of cause and effect relationship between the performance indicators belonging to the Balanced Scorecard (BSC) applied in a hotel. A descriptive, bibliographical, documentary and quantitative methodological approach was used through a case study. Descriptive statistics and analysis of linear models were performed on 14 three-year indicators of a hotel located in João Pessoa - Paraíba (PB), organized based on BSC perspectives: Financial, Clients, Internal Processes and Learning and Growth. It was noticed that indicators of the Customer Perspectives and Learning and Growth influenced the Financial perspective. Indicators of the learning and growth perspective influenced the Internal Processes; Some indicators of Internal Processes influenced the Customers perspective, as well as some indicators from the Customers perspective presented relationships with the Financial perspective, revealing evidence of a cascading effect. It was concluded that there are cause and effect relationships between the indicators studied, but the evidence was not generalized among all indicators. Thus, the study academically contributed to the validation of cause and effect relationships in the BSC perspectives for the hotel sector, as well as in the practice of managers who may have assistance in structuring the BSC and creation of strategic maps.
\end{abstract}

Keywords: Balanced scorecard; cause and effect; hotel sector. 


\section{Introdução}

O crescente desenvolvimento das organizações, em todos os setores, requer que os sistemas e controles estejam evoluindo simultaneamente a esse progresso. Dentro dessa perspectiva de crescimento, as hotelarias estão se destacando cada vez mais. O Serviço Brasileiro de Apoio às Micro e Pequenas Empresas- SEBRAE (2017) verificou que a média brasileira em relação à competitividade do setor hoteleiro está no nível três, de cinco, que é um nível médio de competitividade sobre a área turística, porém com tendência de alcançar o próximo nível de concorrência.

Focando-se em sistemas de controle para acompanhar a evolução das corporações, estão presentes no cotidiano de gestores os métodos de mensuração de desempenho para coordenar e controlar uma empresa. A mensuração de desempenho basicamente é uma ferramenta de quantificação das ações, com o intuito de gerar feedbacks para os gerentes dos diversos setores das organizações (Nelly et al., 1995). Essa técnica permite aos seus usuários identificar as áreas da instituição que estão acima e abaixo da média de produção da empresa, possibilitando a visualização do que deve ser mudado e em qual ponto da entidade devem ocorrer tais mudanças. Um dos sistemas de medição de desempenho mais conhecidos é o Balanced Scorecard (BSC), que foi criado pelos professores de Harvard Robert Kaplan e David Norton. Essa ferramenta foi criada em meados de 1990 e tem como principal objetivo a visualização, por meio de indicadores separados em quatro perspectivas principais (perspectiva Financeira, perspectiva de Clientes, perspectiva de Processos Internos e perspectiva de Aprendizado e Crescimento), das metas da empresa. Todas as perspectivas devem ter relações de causa e efeito entre si, de forma que as ações da empresa tenham no final um retorno na perspectiva Financeira (Kaplan \& Norton, 1992).

A problemática encontrada relacionada ao BSC situa-se na afirmação da existência de relação causa e efeito entre suas perspectivas. Alguns autores, por exemplo, Malina et al. (2007), acreditam que a relação defendida pelos criadores do BSC são simplesmente teóricas e não causais; já outros acreditam que a relação causa e efeito existente é mínima para que possa ser considerada relevante (Silva \& Callado, 2018). Existem também autores que defendem a relação causa e efeito; de forma que consideram a fraca relação causa e efeito um resultado de uma má estruturação do BSC no momento de sua criação (Kaplan \& Norton, 2004).

Considerando o que foi exposto, a questão base que orienta este estudo é: existe relação de causa e efeito entre as perspectivas do BSC? Desta maneira, o objetivo geral do presente artigo é verificar a existência de relação de causa e efeito entre os indicadores de desempenho pertencentes ao Balanced Scorecard (BSC) aplicados em um hotel.

A escolha pelo tema foi influenciada pela significância da discussão controvérsia de um dos pressupostos do BSC, que é a relação de causa e efeito. Dessa forma, o estudo pode contribuir para validação desse pressuposto por meio de verificações empíricas, utilizando de modelos estatísticos baseados nos estudos de Banker et al. (2000) e Bento e Bento (2013), que enfatizaram nos efeitos positivos dos indicadores não financeiros na perspectiva Financeira. Além disso, avança-se com outros testes para mensurar as influências das demais perspectivas do BSC entre si.

A temática sobre causa e efeito tem gerado discussões desde sua criação; e a presente pesquisa contribui empiricamente com resultados testados para os estudos nessa área que, mesmo apresentando casos empíricos, acumula muitos estudos teóricos que não apresentam uma legitimação prática.

A justificativa do campo de análise se deve a necessidade de mais pesquisas serem realizadas no setor hoteleiro brasileiro, visto que estudos nacionais anteriores não apresentaram essa abrangência (Silva \& Callado, 
2018; Vieira \& Callado, 2018). A crescente evolução deste setor na economia brasileira está a se destacar, e, para que seja contínua essa melhora, é necessário que medidas de controle estruturadas corretamente sejam implementadas; por esse motivo o teste empírico é feito para analisar a existência ou não de causa e efeito dos indicadores dentro do BSC do hotel em estudo. Academicamente, também são ainda pioneiros os estudos estatísticos nesse setor do mercado, o que abre espaço para testes e hipóteses a serem estudadas a fim de melhorar os controles hoteleiros.

\section{Referencial teórico}

\subsection{Balanced scorecard}

O BSC é descrito como uma ferramenta de avaliação de desempenho e controle estratégico; também auxilia no momento do alinhamento da organização, uma vez que tem em suas premissas bases o ideal de controle de desempenho em uma relação de cadeia, que é dividida em variáveis de quatro grupos de perspectivas que podem se relacionar entre si. (Mottaghi, 2019)

As perspectivas do BSC são: perspectiva Financeira, perspectiva de Clientes, perspectiva de Processos Internos e perspectiva de Aprendizado e Crescimento (Mateos-Ronco \& Mezquida, 2016). A perspectiva Financeira inclui medidas de desempenho que indicam se as operações feitas pela organização estão reverberando em sua rentabilidade e criação de valor. Tais medidas podem englobar diversos tipos de indicadores, como os de lucratividade, rentabilidade, liquidez, endividamento e outros. Afirma-se que a necessidade de mudança nos indicadores das demais perspectivas do BSC será necessária ou não ao se analisar os resultados obtidos na perspectiva financeira (Kaplan \& Norton, 1992).

A perspectiva de Clientes mostra indicadores de desempenho dos clientes internos e externos, com base na missão da empresa, que estão relacionados ao tempo, à qualidade, ao desempenho e ao custo do bem vendido ou serviço prestado (Kaplan \& Norton, 1992). Normalmente, esses indicadores são obtidos depois de serem feitas algumas entrevistas com os clientes da empresa, abordando temas diversos, como os já mencionados: tempo, qualidade e desempenho; as respostas adquiridas são analisadas para constituição de medidas novas a serem alcançadas a fim de a empresa estar ao máximo atendendo as expectativas (Altaf et al., 2015).

A perspectiva de Processos Internos é a tradução da missão da empresa em atividades internas, a fim de satisfazer os clientes, de forma que cada procedimento feito tenha como fim melhorar os atributos mencionados na perspectiva de Clientes (Kaplan \& Norton, 1992). Tal perspectiva pode estar diretamente relacionada à perspectiva de Clientes, de forma que quando se faz necessário alcançar novas medidas criadas na perspectiva de Clientes, é essencial que os processos internos existentes sejam aprimorados ou que novos procedimentos sejam criados (Garrison et al., 2013).

A perspectiva de Aprendizagem e Crescimento está relacionada aos processos inovadores da empresa e sua capacidade de melhorar continuamente, atendendo as expectativas do mercado, introduzindo produtos e atendimentos que podem criar valor para a empresa ao gerar e evoluir os laços com os clientes (Kaplan \& Norton, 1992). Esta perspectiva está não somente relacionada com a perspectiva de Processos Internos, mas com todos os tipos de avanços de conhecimento que ocorrem na área específica de atuação da empresa. Isso ocorre porque a busca por excelência cresce cada vez mais, e as empresas que não colocarem seus esforços na corrida pelo melhor serviço prestado ou bem vendido ao consumidor não conseguirão manter suas atividades 
estáveis. Dessa forma, quando se encontra um novo conhecimento, a empresa investe na obtenção deste, repercutindo em um melhor processo interno e atendimento ao cliente (Morais et al., 2018).

Garrison et al. (2013) apresentam como as perspectivas do BSC se relacionam, conforme Figura 1. Os autores supracitados ainda enfatizam a ideia dos criadores do BSC de que os indicadores financeiros por si só não podem dar respostas satisfatórias no que diz respeito a caminhos para a melhoria da empresa. Dessa forma, tais bases devem estar integradas com as medidas não financeiras. Ressalta-se que os indicativos financeiros são resultados de ações passadas e os não financeiros são direcionadores que levam, em última análise, a resultados financeiros futuros.

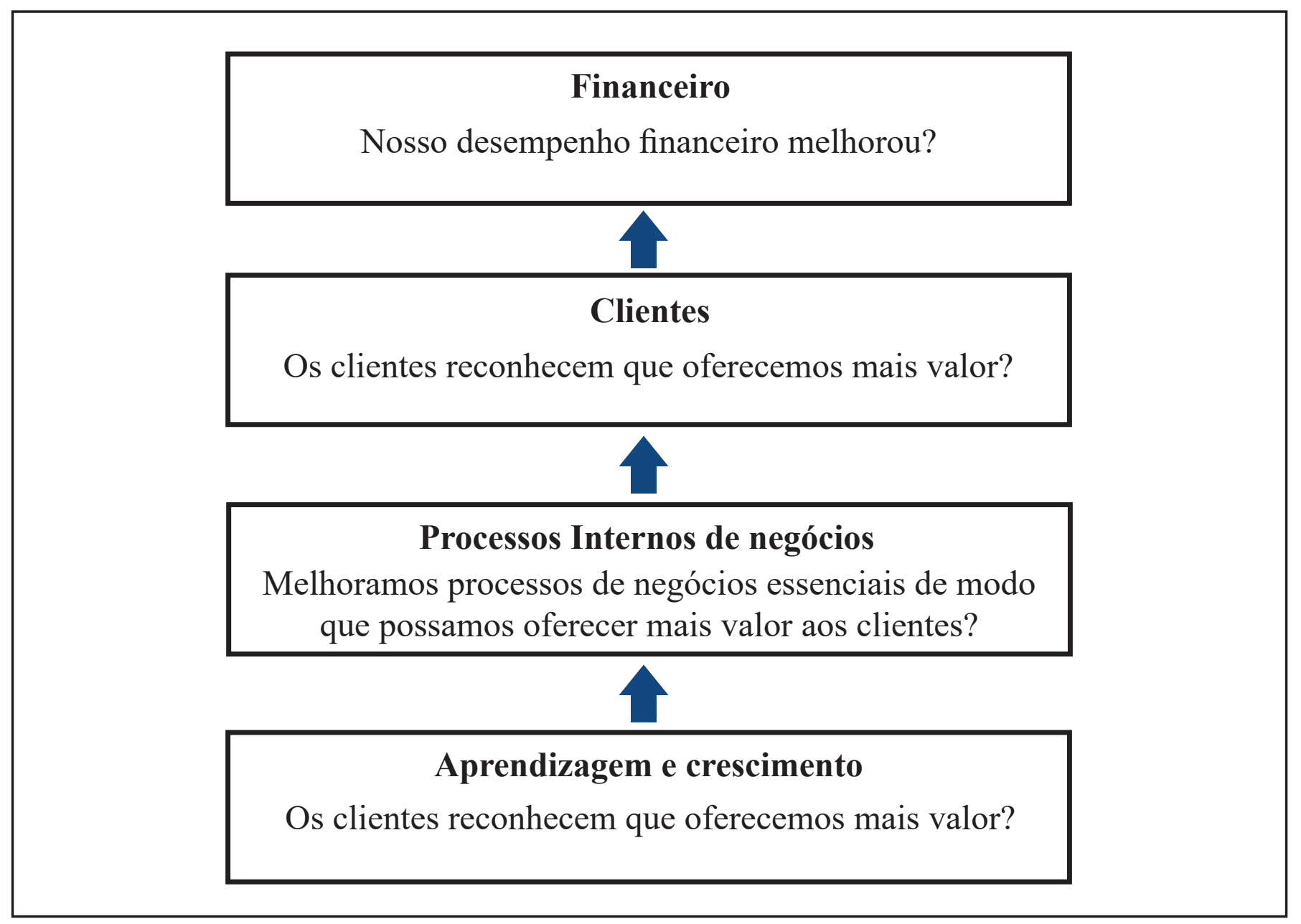

Fig.1. Indicadores de desempenho

Fonte: Adaptado de Garrison et al. (2013, p. 484)

Como mostra a figura 1, de baixo para cima estão todas as perspectivas do BSC, que tendem a possuir relação de causa e efeito. A perspectiva na base da figura mostrada, quando atingida, irá influenciar a segunda acima dela, e assim sucessivamente até que apareçam retornos significativos na perspectiva Financeira (Kaplan \& Norton, 1992).

\subsection{Discussão sobre o tema causa e efeito}

Desde a implementação do BSC nas organizações, têm-se feito muitas críticas a esse modelo de controle de desempenho. A causa dessas críticas é o argumento de que a relação causal defendida pelos seus adeptos não é comprovada empiricamente, e sim por ideias subjetivas. São poucas as pesquisas que visam analisar empiricamente o efeito do uso do BSC nas organizações (Perramon et al., 2015). 
Alguns autores defendem que a relação existente entre os indicadores do BSC é teórica, não causal. Isso significa que as medidas do BSC estão relacionadas apenas de forma argumental, por exemplo, se "a qualidade percebida do produto melhorou (perspectiva Clientes)", então "a receita bruta de vendas aumentará (perspectiva Financeira)" (Malina et al., 2007). Outros defendem que mesmo existindo relação teórica, a relação causal é mínima (Silva \& Callado, 2018).

Os defensores do BSC resguardam a relação causal afirmando que a baixa relação obtida em alguns estudos é resultado de um BSC mal elaborado (Mooraj et al., 1999). A implantação do BSC em uma organização demanda tempo, pois ponderações devem ser feitas para adaptar esta ferramenta de estratégia e controle para cada tipo de empresa. Dessa forma, a falta de planejamento e elaboração ruim do BSC podem resultar em indicadores que não representam fidedignamente as metas a serem atingidas (Modak, Ghosh \& Pathak, 2019).

Com base na discussão apresentada, algumas pesquisas relacionadas ao assunto foram encontradas na literatura (Mooraj et al., 1999; Banker et al., 2000; Norreklit, 2000; Bento \& Bento, 2013; Patzlaff \& Patzlaff, 2015; Porporato et al., 2017; Silva \& Callado, 2018; Vieira \& Callado, 2018).

Mooraj et al. (1999) estudaram a função do BSC dentro de uma organização, verificando se ele é um "bem necessário" ou um "mal não necessário". Os autores neste estudo, além de chegarem ao resultado de que o BSC é um sistema de controle estratégico que oferece aos gestores possibilidades de combinação de sistemas de controle, agregando valor à gestão, consideraram a relação causa e efeito como algo normal dentro do BSC. Defenderam também a importância da escolha de indicadores apropriados na criação desse sistema de controle, pelo fato de que ele tem como ofício transmitir o objetivo financeiro em metas operacionais que deverão ser seguidas pelos colaboradores; o que não permite erros de discernimento na definição de medidas. Esta importância defendida corrobora com pensamentos anteriores que atribuem a falta de causalidade a uma estruturação do BSC mal feita.

Banker et al. (2000) estudaram a mudança no desempenho financeiro em decorrência da implementação de um plano de operação que incluía desempenhos não financeiros em 18 propriedades de uma cadeia de hotéis. $\mathrm{O}$ estudo feito encontrou relações significativas entre as medidas não financeiras com o desempenho financeiro. Além disso, após esse plano, a satisfação do cliente aumentou. Os autores explanaram que o aumento da satisfação do cliente não gera grande aumento no desempenho financeiro atual, mas sim nos futuros. Isso porque essa avaliação feita pelos clientes geraria reconhecimento de mercado crescente, de forma que futuramente mais clientes procurariam seus serviços, elevando assim seus resultados financeiros.

Norreklit (2000) examinou até que ponto existe relação de causa e efeito entre as quatro áreas do BSC, verificando se pode haver vinculação da estratégia às métricas operacionais que os gerentes podem entender e influenciar. O estudo chegou à conclusão de que o BSC não tem uma relação causal, mas sim teórica como já comentado. Seus resultados mostram, por exemplo, que a satisfação do cliente não necessariamente produz bons efeitos financeiros. Assim, de acordo com a autora, o BSC concebe suposições inválidas, podendo fazer com que indicadores indevidos sejam considerados no controle estratégico, repercutindo em um desempenho abaixo do esperado. Consequentemente, não o considera uma ferramenta de gestão estratégica válida, pois não garante um enraizamento organizacional; ainda o considera ineficiente também no enraizamento ambiental, gerando lacunas entre a estratégia planejada e a estratégia evidenciada nas operações da instituição.

Bento e Bento (2013) propuseram e testaram um modelo para verificar e explicar a existência ou não de relação causa e efeito entre as quatro perspectivas do BSC. A pesquisa realizada foi feita usando um modelo de regressão em um banco de dados disponível publicamente de 332 empresas incluídas no American 
Institute of Certified Public Accountants Performance Measurement Survey. O estudo feito encontrou relação diretamente proporcional entre as perspectivas base do BSC com as suas sucessoras em uma relação cascata, corroborando com a ideia defendida por Mooraj et al. (1999).

Patzlaff e Patzlaff (2015) investigaram, também, através do uso da estatística, a existência ou não de relação causa e efeito entre as variáveis pertencentes ao BSC em uma entidade do Terceiro Setor. O resultado obtido na análise feita pelos pesquisadores corroborou com a premissa da existência de relação entre as variáveis de cada perspectiva do BSC sustentada pelos autores seminais Kaplan e Norton (1992).

Porporato et al. (2017) estudaram de forma direcionada a verificar a existência ou não de correlações significativas entre medidas de Processos Internos e Aprendizado e Crescimento e os resultados relativos nas perspectivas Clientes e Financeira dentro do BSC de um hospital da comunidade de Ontaro, Canadá. Tal estudo foi feito entre os anos de 2010 e 2012, as conclusões publicadas em 2017. Os resultados obtidos evidenciaram relações causa e efeito macros na estrutura geral do BSC, porém não conseguiu explicar a não relação de influência micro em algumas variáveis dentro do esqueleto do scorecard. Vale mencionar, porém, que fizeram a observação de que a estrutura da ferramenta de controle criada pela organização poderia ou não influir nos resultados obtidos de relação causal, concordando com o que Mooraj et al (1999) mencionam em suas conclusões.

Nacionalmente, Silva e Callado (2018) tiveram como objetivo de estudo também a verificação da existência ou não da relação causa e efeito evidenciada na literatura entre as variáveis do BSC. Seus estudos foram aplicados em uma distribuidora de combustível. Utilizando-se de correlação de Pearson e regressão linear, chegaram à conclusão de que as relações existentes eram fracas ou moderadas. Para os autores especificados, a utilização de correlação e regressão poderia ser útil não somente na verificação da relação causa e efeito quando um BSC já estivesse feito, mas também no momento de sua criação.

Por fim, Vieira e Callado (2018) estudaram e analisaram a significância estatística da linearidade e causalidade das relações entre os indicadores do BSC. Para tal, manusearam dados de 15 indicadores referentes a 31 meses de informações obtidas e mensuradas internamente por uma empresa de consultoria do nordeste brasileiro; além de usar os métodos estatísticos correlação de Pearson e regressão linear. A análise feita apresentou evidências de interligações entre os indicadores das diferentes perspectivas, porém em apenas parte deles, de maneira que outros indicadores não apresentavam nenhuma relação aparente entre si, o que, para eles, pode tratar-se de problemas na seleção de indicadores pela empresa; tal pensamento condiz com o exposto pelos autores Porporato et al. (2017).

Com base em todas as informações apresentadas, o presente estudo tem como objetivo testar a seguinte hipótese:

H0: Existe relação de causa e efeito entre os indicadores de desempenho pertencentes ao BSC no âmbito de um hotel.

\section{Metodologia}

O primeiro aspecto metodológico se refere ao delineamento da pesquisa. Dessa forma, foi considerada uma abordagem metodológica de natureza descritiva, bibliográfica e documental, aplicando-se uma abordagem quantitativa através de um estudo de caso. Ruiz (2006) define pesquisa bibliográfica como o estudo dos trabalhos já feitos em determinada área, para levantamento e análise do que já se produziu. Assim posterioriormente descrever o conhecimento já alcançado sobre o tema. 
A pesquisa documental, diferente da pesquisa bibliográfica, busca material não editado, por exemplo cartas, relatórios, avaliações, etc. Os dados obtidos subsidiam o melhor entendimento sobre o objeto estudado e possibilitam a confiabilidade de achados por meio de outras fontes (Martins \& Theóphilo, 2007).

O estudo de caso trata-se de uma averiguação prática que pesquisa os fenômenos dentro de seu contexto real. Nesse caso o pesquisador não tem controle sobre os eventos e os resultados obtidos, podendo somente os analisar, descrever e inferir da maneira mais precisa possível esses resultados (Martins \& Theóphilo, 2007).

O segundo aspecto metodológico corresponde à definição da unidade de análise da pesquisa. Assim, o estudo de caso foi realizado em um hotel localizado na cidade de João Pessoa- Paraíba (PB). O hotel foi inaugurado em 2008, com o lema de sustentabilidade e conforto. Apresenta diversos prêmios de qualidade como o certificado de excelência TripAdvisor, que é concedido aos estabelecimentos do setor de turismo e hotelaria no mundo que prestam serviços de alta qualidade (TripAdvisor, 2018).

O terceiro aspecto metodológico foi a coleta de dados. Esse processo se deu por solicitação documental dos indicadores de desempenho utilizados pela empresa. A empresa disponibilizou dois tipos de relatórios gerenciais, o primeiro, intitulado "Gerenciamento de Rotina" e o segundo, intitulado "Indicadores Gerenciais", no período de 36 meses, compreendendo os anos de 2016 a 2018. Após isso, tabulou-se um total de 14 indicadores de mensuração de desempenho com base nas quatro perspectivas do BSC (Kaplan \& Norton, 1992), que apresentaram informações completas no período analisado, conforme Quadro 1.

\section{Quadro 1}

Indicadores de desempenho nas perspectivas do BSC

\begin{tabular}{|c|c|c|}
\hline PERSPECTIVA & \multicolumn{2}{|c|}{ INDICADORES } \\
\hline \multirow{2}{*}{ FINANCEIRA } & FIN_IRB & Índice de Receita Bruta \\
\hline & FIN_IDMÉDIA & Índice de Diária Média \\
\hline \multirow{5}{*}{ CLIENTES } & CLIENT_ISCE & $\begin{array}{l}\text { Índice de Satisfação dos Clientes com } \\
\text { Eventos }\end{array}$ \\
\hline & CLIENT_ISCCB & $\begin{array}{l}\text { Índice de Satisfação do Cliente com o } \\
\text { Coffee Break }\end{array}$ \\
\hline & CLIENT_ISCG & $\begin{array}{l}\text { Índice de Satisfação do Cliente com a } \\
\text { Governança }\end{array}$ \\
\hline & CLIENT_ISCR & $\begin{array}{l}\text { Índice de Satisfação do Cliente com a } \\
\text { Recepção }\end{array}$ \\
\hline & CLIENT_ISCSR\&C & $\begin{array}{l}\text { Índice de Satisfação do Cliente com o } \\
\text { Serviço de Restaurante \& Cozinha }\end{array}$ \\
\hline \multirow{3}{*}{ PROCESSOS INTERNOS } & PROCESS_ICASD & $\begin{array}{l}\text { Índice de Cumprimento das Ações de } \\
\text { Segurança dos Dados (Rede, Anti Vírus, } \\
\text { Backup) }\end{array}$ \\
\hline & PROCESS_IUHs & $\begin{array}{l}\text { Índice de UHs Limpas e Arrumadas } \\
\text { Corretamente até às 16:00h }\end{array}$ \\
\hline & PROCESS_IAPMAC & $\begin{array}{l}\text { Índice de Atendimento dos Prazos das } \\
\text { Manutenções Corretivas }\end{array}$ \\
\hline \multirow{4}{*}{ APRENDIZAGEM/CRESCIMENTO } & APRENeCRESC_IAFNP & $\begin{array}{l}\text { Índice de Acompanhamentos Funcionais } \\
\text { de Novatos e Promovidos }\end{array}$ \\
\hline & APRENeCRESC_ITREIN & Índice de Treinamentos Realizados \\
\hline & APRENeCRESC_IQTREIN & $\begin{array}{l}\text { Índice de Satisfação com a Qualidade } \\
\text { dos Treinamentos }\end{array}$ \\
\hline & APRENeCRESC_ITURNOVER & Índice de Turnover \\
\hline
\end{tabular}

Ressalta-se que todos os indicadores de desempenho são acompanhados mensalmente e mensurados 
por meio de percentuais de atendimento das metas estratégicas. Essas metas e respectivos sinais esperados serão apresentados na Tabela 1, que representa o procedimento inicial do quarto aspecto da metodologia, as técnicas de análise dos resultados.

Para análise desta pesquisa foi utilizada a técnica estatística descritiva, por meio de medidas de tendência central e dispersão, bem como modelos de regressões lineares múltiplas, estimadas por mínimos quadrados ordinários (OLS), para verificar a existência de relação de causa e efeito entre os indicadores de desempenho pertencentes ao BSC, fundamentando-se nos estudos de Banker et al. (2000), Bento e Bento (2013) e Vieira e Callado (2018).

Visando responder a hipótese central do estudo, utilizaram-se inicialmente modelos lineares de primeira ordem das perspectivas do BSC, esperando-se encontrar a influência positiva dos indicadores não financeiros na perspectiva Financeira (Banker et al., 2000; Bento \& Bento, 2013), com base nas equações da Tabela 2. De forma complementar, utilizou-se modelos lineares de segunda ordem das perspectivas do BSC, esperando o impacto direto entre si, na seguinte sequência teórica de influência: perspectiva de Aprendizado e Crescimento $\rightarrow$ perspectiva de Processos Internos $\rightarrow$ perspectiva de Clientes $\rightarrow$ perspectiva Financeira (Kaplan \& Norton, 1992; Vieira \& Callado, 2018), conforme Tabelas 3 e 4.

\section{Análise e discussão dos resultados}

\subsection{Estatísticas descritivas}

A Tabela 1 apresenta as estatísticas descritivas dos indicadores de desempenho utilizados pela empresa objeto de análise, no período investigado.

\section{Tabela 1}

Estatísticas descritivas

\begin{tabular}{|l|c|c|c|c|c|}
\hline \multicolumn{1}{|c|}{ Variáveis } & Metas & Sentidos & Média & Desv.P & Mínimo \\
\hline FIN_IRB & $100 \%$ & & 0,9401 & 0,1326 & 0,59 \\
\hline FIN_IDMÉDIA & $100 \%$ & & 0,9089 & 0,1086 & 0,6523 \\
\hline CLIENT_ISCE & $96 \%$ & & 0,9527 & 0,0273 & 0,89 \\
\hline CLIENT_ISCCB & $93 \%$ & & 0,9253 & 0,0463 & 0,79 \\
\hline CLIENT_ISCG & $92 \%$ & & 0,9216 & 0,0127 & 0,90 \\
\hline CLIENT_ISCR & $95 \%$ & & 0,9702 & 0,0120 & 0,95 \\
\hline CLIENT_ISCSR\&C & $92 \%$ & & 0,9230 & 0,0158 & 0,90 \\
\hline PROCESS_ICASD & $100 \%$ & & 0,9994 & 0,0286 & 0,83 \\
\hline PROCESS_IUHs & $100 \%$ & & 0,9991 & 0,0027 & 0,9855 \\
\hline PROCESS_IAPMAC & $95 \%$ & & 0,9778 & 0,0258 & 0,89 \\
\hline APRENeCRESC_IAFNP & $100 \%$ & & 0,9658 & 0,0988 & 0,66 \\
\hline APRENeCRESC_ITREIN & $90 \%$ & & 0,8987 & 0,2191 & 0,12 \\
\hline APRENeCRESC_IQTREIN & $90 \%$ & & 0,9786 & 0,0340 & 0,90 \\
\hline APRENeCRESC_ITURNOVER & $2 \%$ & & 0,0280 & 0,0177 & 0,00 \\
\hline
\end{tabular}

Inicialmente, observa-se que os sentidos de todas as metas, exceto a última, são com sentidos crescentes. Os dados obtidos estão em sua maioria em torno de 0 e 1 , isso porque os indicadores coletados têm como metas os valores que podem variar de $2 \%$ a $100 \%$, respectivamente. Os dois indicadores financeiros não atingiram as metas estratégicas determinadas no BSC no período analisado.

O indicador FIN_IRB teve como média 0,9401; seus valores mínimo e máximo foram aproximadamente 
0,59 e 1,1116, respectivamente. É relevante mencionar que alguns indicadores ultrapassaram o valor um (1), isso porque esse resultado corresponde a uma meta estabelecida pela empresa; assim, alguns indicadores podem ultrapassar esse valor. Considerando o desvio padrão do indicador já citado (aproximadamente 13\%), vê-se uma variação entre os anos que pode ser considerada normal, sabendo que essa medida de Receita Bruta pode ter sido resultado da sazonalidade desse setor, uma vez que existem períodos do ano em que a procura por hotéis é maior e vice-versa.

O indicador FIN_IDMEDIA tem como média 0,9089. Seus valores mínimo e máximo são 0,6523 e 1,096, respectivamente. A observação em relação ao desvio padrão deste indicador (aproximadamente 11\%) é semelhante à do primeiro já apresentado.

Os demais indicadores tabulados, que estão nas perspectivas de Clientes, Processos Internos e Aprendizado e Crescimento, apresentam médias todas acima de 0,89, o que significa que a empresa estudada, nos três anos, apresentou bom rendimento em relação aos seus procedimentos. Excetua-se desse intervalo o indicador APRENeCRESC_ITURNOVER, que tem como meta 0,02, visto que é esperado que a rotavidade de funcionários seja decrescente.

Todos os indicadores de Clientes estiveram acima de 0,9. Dos cinco indicadores, os três últimos alcançaram a meta durante o período estudado, dando destaque ao indicador CLIENT_ISCR que teve média 0,9702, com mínimo 0,95 e máximo 0,99. Tais números mostram que a hotelaria, no período estudado, apresentou um bom primeiro contato com seus clientes. O menor indicador dessa perspectiva foi o CLIENT_ ISCSR\&C, com media 0,923, mínimo 0,9 e máximo 0,96 , algo que não é muito preocupante, pois a meta estabelecida foi atingida, porém destaca-se, uma vez que é prezada a excelência nos serviços de gastronomia.

Os três indicadores da perspectiva de Processos Internos apresentaram resultados acima de 0,97, o que pode ser importante para o hotel de estudo. Os dois primeiros tiveram resultados próximos a meta estabelecida, e o último atingiu a meta criada. Dá-se foco ao indicador PROCESS_ICASD, que apresentou media 0,9994, mínimo 0,83 e máximo 1 , mostrando a preocupação que o hotel tem pela segurança de seus dados e dos dados dos clientes. O indicador PROCESS_IUHs também apresentou resultado significativo, com media 0,9991, mínimo 0,9855 e máximo 1 . Seu resultado e sua pequena variação entre mínimo e máximo transmitem a importância dada pelo hotel à entrega de unidades habitacionais bem tratadas a seus clientes.

Entre os indicadores de Aprendizado e Crescimento, somente um dos quatro atingiu a meta estabelecida. A medida APRENeCRESC_ITREIN teve menor média entre os indicadores com sentido de meta crescente, 0,8987, quase alcançando sua meta. Essa medida teve desvio padrão de $21 \%$, o que assinala variações relevantes entre os anos do número de treinamentos feitos durante os anos de dados investigados. Tais variações podem ter sido resultado do aumento de treinamentos realizados desde o primeiro ano estudado. $\mathrm{O}$ indicador da perspectiva de Aprendizado e Crescimento que chama mais atenção é o APRENeCRESC_ITURNOVER, isso porque seu resultado é pequeno, média 0,028 , efeito que se justifica pelo fato de que o sentido da meta para esse indicador decrescente, significando quanto menor melhor. Não obstante, ainda não está dentro da meta estabelecida pelo hotel.

\subsection{Modelos lineares de primeira ordem das perspectivas do BSC}

Os dados obtidos na pesquisa foram testados em um modelo de regressões lineares. Os resultados alcançados estão evidenciados na Tabela 2. 
Tabela 2

Equações que mensuram os efeitos das perspectivas do BSC na perspectiva Financeira

\begin{tabular}{|c|c|c|}
\hline Variáveis & FIN_IRB & FIN_IDMÉDIA \\
\hline \multirow[t]{2}{*}{ CLIENT_ISCE } & $-0,7075$ & 1,4634 \\
\hline & $(1,5458)$ & $(1,1506)$ \\
\hline \multirow[t]{2}{*}{ CLIENT_ISCCB } & 1,2973 & 0,1644 \\
\hline & $(0,8082)$ & $(0,6167)$ \\
\hline \multirow[t]{2}{*}{ CLIENT_ISCG } & $-0,9489$ & $-3,9586 * *$ \\
\hline & $(2,2836)$ & $(1,6999)$ \\
\hline \multirow[t]{2}{*}{ CLIENT_ISCR } & $-0,2387$ & 0,2493 \\
\hline & $(2,0338)$ & $(1,5139)$ \\
\hline \multirow[t]{2}{*}{ CLIENT_ISCSR\&C } & 1,1666 & 1,6505 \\
\hline & $(1,7181)$ & $(0,6103)$ \\
\hline \multirow[t]{2}{*}{ PROCESS_ICASD } & 0,1457 & 0,0120 \\
\hline & $(0,8200)$ & $(0,6103)$ \\
\hline \multirow[t]{2}{*}{ PROCESS_IUHs } & $-10,1532$ & $-5,1858$ \\
\hline & $(8,3303)$ & $(6,2009)$ \\
\hline \multirow[t]{2}{*}{ PROCESS_IAPMAC } & 1,2397 & 0,8332 \\
\hline & $(0,9862)$ & $(0,7341)$ \\
\hline \multirow[t]{2}{*}{ APRENeCRESC_IAFNP } & $-0,3230$ & $-0,2514$ \\
\hline & $(0,2501)$ & $(0,1862)$ \\
\hline \multirow[t]{2}{*}{ APRENeCRESC_ITREIN } & $-0,0031$ & $-0,1003$ \\
\hline & $(0,1169)$ & $(0,0870)$ \\
\hline \multirow[t]{2}{*}{ APRENeCRESC_IQTREIN } & $-1,7361 * *$ & $-0,4453$ \\
\hline & $(0,7927)$ & $(0,5901)$ \\
\hline \multirow[t]{2}{*}{ APRENeCRESC_ITURNOVER } & $-3,1539 * *$ & $-2,3945 * *$ \\
\hline & $(1,4567)$ & $(1,0844)$ \\
\hline \multirow[t]{2}{*}{ Const } & 11,3334 & 6,4363 \\
\hline & $(8,1096)$ & $(6,0366)$ \\
\hline Teste F & 0,1109 & 0,0249 \\
\hline $\mathrm{R}^{2}$ & 0,4831 & 0,5730 \\
\hline $\mathrm{R}^{2}$ ajustado & 0,2134 & 0,3502 \\
\hline Obs. & 36 & 36 \\
\hline
\end{tabular}

**indica significância de 5\%, respectivamente. Erro-padrão de Newey-West entre parênteses.

Observando a tabela acima, pode-se verificar que em uma visão geral, poucas perspectivas tiveram influência nas perspectivas Financeiras. O indicador CLIENT_ ISCG teve influência $(-3,9586)$ sobre o indicador financeiro FIN_IDMÉDIA e o indicador APRENeCRESC_IQTREIN teve influência $(-1,7361)$ sobre o indicador financeiro FIN_IRB. Ambas as relações tiveram significância de 5\%. Essas relações inversamente proporcionais encontradas chamam atenção pelo fato de que a teoria encontrada sobre esses indicadores (Kaplan \& Norton, 1992) indica que a relação normalmente deveria ser diretamente proporcional. Resultados semelhantes sobre relações inversamente proporcionais são encontradas no trabalho de Rabo (2014). Não sendo encontradas na literatura sobre hotéis resultados semelhantes, levanta-se a possibilidade de tal resultado ter sido atingido pela eventualidade de mesmo com a aprovação dos clientes em relação à gerencia, aspectos externos às vontades daqueles fizeram com que estes tivessem resultados menores.

O indicador APRENeCRESC_ITURNOVER teve influência nos dois indicadores financeiros, FIN_ IRB e FIN_IDMÉDIA, com resultados -3,1539 e -2,3945, nesta ordem. Essas influências tiveram significância 
média também de 5\%. Vale relatar que nesse indicador, o resultado foi condizente com o esperado, pois o turnover considerado bom para uma empresa deve ter relação inversamente proporcional com os indicadores financeiros, visto que, ao reduzir a rotatividade de funcionários, as metas financeiras tendem a ser alcançadas com mais facilidades.

\subsection{Modelos lineares de segunda ordem das perspectivas do BSC}

Abaixo serão evidenciadas tabelas com os demais resultados encontrados, agora em relação à influência entre as perspectivas de segunda ordem do BSC.

\section{Tabela 3}

Equações que mensuram os efeitos da perspectiva Aprendizado e Crescimento na perspectiva de Processos Internos

\begin{tabular}{|c|c|c|c|}
\hline Variáveis & PROCESS_ICASD & PROCESS_IUHs & PROCESS_IAPMAC \\
\hline \multirow[t]{2}{*}{ APRENeCRESC_IAFNP } & $-0,0285$ & $-0,0037$ & 0,0247 \\
\hline & $(0,0531)$ & $(0,0049)$ & $(0,0415)$ \\
\hline \multirow[t]{2}{*}{ APRENeCRESC_ITREIN } & $-0,0136$ & $-0,0016$ & $0,0568 * * *$ \\
\hline & $(0,0241)$ & $(0,0022)$ & $(0,0188)$ \\
\hline \multirow[t]{2}{*}{ APRENeCRESC_IQTREIN } & $-0,0793$ & $-0,0051$ & 0,1800 \\
\hline & $(0,1624)$ & $(0,0152)$ & $(0,1271)$ \\
\hline \multirow[t]{2}{*}{ APRENeCRESC_ITURNOVER } & 0,0226 & 0,0113 & $0,4970 * *$ \\
\hline & $(0,3063)$ & $(0,0288)$ & $(0,0239)$ \\
\hline \multirow[t]{2}{*}{ Const } & $1,1113 * * *$ & $1,0089 * * *$ & $0,7126^{* * *}$ \\
\hline & $(0,1867)$ & $(0,0175)$ & $(0,1461)$ \\
\hline Teste $\mathrm{F}$ & 0,9467 & 0,8526 & 0,0417 \\
\hline $\mathrm{R}^{2}$ & 0,0228 & 0,0414 & 0,2670 \\
\hline $\mathrm{R}^{2}$ ajustado & $-0,1033$ & $-0,0823$ & 0,1724 \\
\hline Obs. & 36 & 36 & 36 \\
\hline
\end{tabular}

** indica significância de 5\%,***indica significância de 1\%, respectivamente. Erro-padrão de Newey-West entre parênteses.

Observando os dados da Tabela 3, é possível visualizar que os indicadores APRENeCRESC_ITREIN e APRENeCRESC_ITURNOVER influenciaram a medida PROCESS_IAPMAC, com resultados de 0,0568 (significância de 10\%) e 0,4970 (significância de 5\%), respectivamente. A relação diretamente proporcional encontrada na primeira ligação mostrada corrobora com os resultados dos autores sobre o tema da relação causa e efeito em hotéis (Banker et al., 2000), de que o aumento em um indicador faz com que haja avanço nos resultados de indicadores seguintes na relação cascata do BSC. A relação diretamente proporcional, encontrada na segunda dependência, não é normalmente encontrada, uma vez que uma rotatividade maior poderia diminuir a eficácia de alguns procedimentos. Contudo, os resultados indicam que o treinamento feito com os novos funcionários pode incentivar o bom rendimento nos procedimentos realizados; assim, mesmo com rotatividade, ocorreria um maior alcance nos procedimentos de Processos Internos.

\section{Tabela 4}

Equações que mensuram os efeitos da perspectiva de Processos Internos na perspectiva de Clientes

\begin{tabular}{lccccc}
\hline Variáveis & CLIENT_ISCE & CLIENT_ISCCB & CLIENT_ISCG & CLIENT_ISCR & CLIENT_ISCSR\&C \\
\hline PROCESS_ICASD & $-0,2925$ & $-0,4921$ & $-0,1669^{* *}$ & $-0,0528$ & $-0,0843$ \\
& $(0,1587)$ & $(0,2747)$ & $(0,0693)$ & $(0,0733)$ & $(0,0953)$ \\
PROCESS_IUHs & $-0,8754$ & $-0,1501$ & $1,2986^{*}$ & 0,8040 & 0,7017 \\
& $(1,6603)$ & $(2,8709)$ & $(0,7247)$ & $(0,7668)$ & $(0,9975)$
\end{tabular}




\begin{tabular}{|c|c|c|c|c|c|}
\hline \multirow{2}{*}{$\begin{array}{l}\text { PROCESS } \\
\text { IAPMAC }\end{array}$} & 0,2460 & 0,2036 & 0,0721 & 0,0006 & 0,1062 \\
\hline & $(0,1757)$ & $(0,3040)$ & $(0,0767)$ & $(0,0811)$ & $(0,1055)$ \\
\hline \multirow[t]{2}{*}{ Const } & 1,8778 & 1,3655 & $-0,2805$ & 0,2187 & 0,2019 \\
\hline & $(1,6718)$ & $(2,8928)$ & $(0,7297)$ & $(0,7721)$ & $(1,0044)$ \\
\hline Teste F & 0,2033 & 0,3489 & 0,0292 & 0,6345 & 0,5269 \\
\hline $\mathrm{R}^{2}$ & 0,1321 & 0,0963 & 0,2421 & 0,0513 & 0,0662 \\
\hline $\mathrm{R}^{2}$ ajustado & 0,0507 & 0,0116 & 0,1711 & $-0,0377$ & $-0,0213$ \\
\hline Obs. & 36 & 36 & 36 & 36 & 36 \\
\hline
\end{tabular}

*indica significância de 10\% e** indica significância de 5\%, respectivamente. Erro-padrão de Newey-West entre parênteses.

Contemplando a Tabela 4, pode-se visualizar que um único indicador da perspectiva de Clientes foi influenciado. A medida CLIENT_ISCG foi influenciada pelos indicadores PROCESS_ICASD e PROCESS IUHs com resultados -0,1669 (a nível de significância de 5\%) e 1,2986 (a nível de significância de 10\%), respectivamente. A influência inversamente proporcional encontrada nessa tabela (entre PROCESS_ICASD e CLIENT_ISCG) é controvérsia, considerando que esse resultado é diferente do esperado e encontrado nos estudos de Kaplan e Norton (1992) e Banker et al (2000). Contudo, tal resultado corrobora no que diz respeito à discussão da relação causa e efeito dos Processos Internos na perspectiva de Clientes, verificada no estudo de Rabo (2014). A relação diretamente proporcional encontrada (entre PROCESS_IUHs e CLIENT_ ISCG) corrobora com os estudos já acima citados: Kaplan e Norton (1992) e Banker et al (2000); tal resultado manifesta a ideia de que um bom tratamento das unidades habitacionais dentro dos hotéis faz com que seus clientes melhorem sua visão sobre a governança.

\section{Considerações finais}

O objetivo geral do estudo foi verificar a existência da relação causa e efeito entre os indicadores de desempenho pertencentes ao BSC no âmbito de um hotel. A motivação para tal escolha foi a discussão existente sobre o tema causa e efeito desde a criação do balanced scorecard até os dias atuais. Para tal, foi utilizada uma abordagem metodológica de maneira descritiva, bibliográfica e documental aplicando-se uma abordagem quantitativa através de um estudo de caso.

O estudo de caso foi realizado em um hotel localizado na cidade de João Pessoa - PB. As informações obtidas foram organizadas em 14 indicadores com base nas quatro perspectivas do BSC (Kaplan \& Norton, 1992); e foram utilizadas estatísticas descritivas e modelos de regressão lineares múltiplas, estimadas por mínimos quadrados ordinários (OLS), para verificar a existência de relação causa e efeito entre os indicadores de desempenho.

No que se refere aos resultados encontrados das relações causa e efeito entre os indicadores de desempenho, verificou-se que dos 12 indicadores das perspectivas de primeira ordem, três deles tiveram influencias significativas dentro das duas perspectivas Financeiras, sendo um da perspectiva de Clientes e dois da perspectiva de Aprendizado e Crescimento. Considerando a análise entre a relação dos indicadores de segunda ordem, dois dos quatro indicadores de Aprendizado e Crescimento tiveram influência em um indicador da perspectiva de Processos Internos; e dois indicadores, de três, da perspectiva de Processos Internos tiveram influência em um indicador da perspectiva de Clientes. Enfatizam-se ainda as relações inversamente proporcionais encontradas, diferentes do que normalmente se espera e encontra na literatura base sobre o tema. 
Isto posto, o presente estudo obteve êxito em responder o problema de pesquisa. A hipótese central da pesquisa (H0) não pode ser rejeitada, pois existe relação de causa e efeito entre os indicadores de desempenho pertencentes ao BSC no âmbito de um hotel, mesmo que somente entre alguns indicadores, conforme estudo anterior (Vieira \& Callado, 2018). Os autores Kaplan e Norton (2004) sugerem que uma relação fraca entre os indicadores de desempenho é proveniente de uma má estruturação do BSC, uma vez que a elaboração deste controle de desempenho requer conhecimento prévio dos indicadores existentes na empresa e compreensão de possíveis medidas a serem criadas para atender as relações que se esperam depois de criado o BSC.

O presente estudo tem relevância teórica no que diz respeito ao estudo das relações de causa e efeito dentro do Balanced Scorecard, principalmente no setor hoteleiro. Os estudos realizados nacionalmente ainda são iniciais, com poucos setores contemplados. Assim, esse estudo também servirá como referencial para estudos futuros. De maneira prática, a presente pesquisa atua como auxiliadora no processo de organização e criação da estratégia, ao evidenciar indicadores com relações de causa e efeito.

O setor hoteleiro, como qualquer outro, necessita de controles mais estruturados e testados empiricamente, em razão da competitividade entre hotéis estar em fase de crescimento em nosso país; e esse artigo teve como intuito a criação de informações relevantes a serem consideradas no momento da criação dos controles de desempenho das organizações, sugerindo a criação do BSC e de mapas estratégicos considerando relações de causalidade testadas empiricamente.

Importante informar que ocorreram limitações metodológicas, visto que os 14 indicadores mostrados foram os únicos constantes durante os três anos estudados. Outros indicadores foram disponibilizados pelo hotel, porém sem constância de aparecimento entre os anos, o que dificultou a análise de relação em um período mais elevado. Destaca-se também a escassez de trabalhos nacionais para comparação com o estudo atual, de forma que não se tem ainda uma base de resultados abrangentes para comparação de resultados de BSCs hoteleiros nacionais. Por fim, os resultados não podem ser generalizados para outros setores, pois a pesquisa se tratou de um estudo de caso. Sugere-se para estudos futuros o uso da relação causa e efeito também no momento da criação do BSC em outros setores nacionais.

\section{Referências}

Altaf, J. G. et al.( 2015). Clínica Olhar: Análise da Satisfação dos Clientes em Relação ao Serviço Prestado. RASI, Volta Redonda, v. 1, n. 1, p. 21-36.

Banker, R. D., Potter, G., Srinivasan, D. (2000). An Empirical Investigation of an Incentive Plan That Includes Nonfinancial Performance Measures. American Accounting Association, v. 75, n. 1, p. 65-92.

Bento, A., Bento, R. (2013). Validating Cause-and-Effect Relationships in the Balanced Scorecard. Academy of Accounting and Financial Studies Journal, v. 17, n. 3, p. 45-55.

Castro, José Roberto. (2017). Como a crise afetou o cidadão comum em 2016.Nexo Jornal. Disponível em: $<$ https://www.nexojornal.com.br/expresso/2017/01/14/Como-a-crise-afetou-o-cidad\%C3\%A3o-comumem-2016>. Acesso em 20 de Julho de 2019. 
Garrison, R. H., Noreen, E. W., Brewer, P. C. (2013). Contabilidade Gerencial. 14. ed. AMGH, Porto Alegre.

Kaplan, R. S., Norton, D. P. (1992). The Balanced Scorecard - Measures that Drive Performance. Harvard Business Review, v. 1, n. 1, p. 1-11.

Kaplan, R. S., Norton, D. P. (2004). Putting the Balanced Scorecard to Work. Harvard Business Review, n. 2, p. 2-19.

Malina, M. A., Norreklit, H. S. O., Selto, F. H. (2007). Relations among Measures, Climate of Control, and Performance Measurement Models. Contemporary Accounting Research, v. 24, n. 3, p. 935-982.

Martins, G. D. A.; Theóphilo, C. R. (2007). Metodologia da Investigação Científica para Ciências Sociais Aplicadas. Atlas ,São Paulo.

Mateos-Ronco, A.; Mezquida, J. M. H. (2016). Developing a performance management model for the implementation of TQM practices in public education centres. Total Quality Management \& Business Excellence, v. 29, n. 5-6, p. 546-579.

Modak, M.; Ghosh, K. K.; Pathak, K. (2019). A BSC-ANP approach to organizational outsourcing decision support-A case study. Journal of Business Research, v. 103, p. 432-447.

Mooraj, S., Oyon, D., Hostettler, D. (1999). The Balanced Scorecard: a Necessary Good or an Unnecessary Evil? European Management Journal, v. 17, n. 5, p. 481-491.

Morais, M. D. O. et al. (2018). Organização para inovação nas empresas: proposta de modelo integrando estratégia e estrutura organizacional - estudo de caso em uma empresa de borracha. Brazilian Journal of Development, Curitiba, v. 4, n. 5, Edição Especial, p. 2503-2521.

Mottaghi, M. (2019). Improving organizational performance along with strategic planning in the framework of combining the david model and bsc assessment model. International Journal of Information, Business and Management, v. 11, n. 2, p. 256-270.

Nelly, A., Gregory, M., Platts, K. (1995). Performance measurements system design: a literature review andresearch agenda, v. 15 , n. 4, p. 80-116.

Norreklit, H. (2000). The balance on the balanced scorecard - a critical analysis of some of its assumptions. Management Accounting Research, v. 11, n. 1, p. 65-88.

Patzlaff, A. C., Patzlaff, P. M. G. (2015). Análise da relação “causa e efeito" do balanced scorecard: um estudo aplicado no terceiro setor. Faro, Valparaíso, v. 1, n. 21, p. 3-25. 
Perramon, J. et al. (2015). Learning to create value through the 'balanced scorecard' model: an empirical study. Total Quality Management \& Business Excellence, v. 27, n. 9-10, p. 1121-1139.

Porporato, M., Tsasis, P., Vinuesa, L. M. M. (2017). Do hospital balanced scorecard measures reflect causeeffect relationships? International Journal of Productivity and Performance Management, 66, n. 3, 338-361.

Rabo, J. (2014). A Study on Effect of Non-Financial Balanced Scorecard Perspectives on Financial Performance of Property Firms Listed in the Philippine Stock Exchange. Journal of Global Business, v. 3, n. 1, p. 13-18.

Ruiz, J. Á. (2006). Metodologia Científica: Guia para Eficiência nos Estudos. 6. ed. Atlas ,São Paulo.

Sebrae Inteligência Setorial. Dimensões de competitividade no turismo - monitoramento e infraestrutura. Disponível em: <https://sebraeinteligenciasetorial.com.br/produtos/relatorios-de-inteligencia/dimensoes-decompetitividade-no-turismo-monitoramento-e-infraestrutura/59b682d93055761900174658>. Acesso em 15 de Julho de 2019

Silva, A. M. G. D., Callado, A. A. C. (2018). Análise da relação “causa-efeito” do Balanced Scorecard (BSC): Um estudo de caso aplicado em uma empresa distribuidora de combustível. In: XXV Congresso Brasileiro de Custos. Vitória: [s.n.].

Tripadvisor. Certificado de Excelência. Disponível em: $<$ https://www.tripadvisor.com.br/>.Acesso em: 19 de julho de 2019.

Vieira, L. M. E. D. R., Callado, A. A. C. (2018). Relações de causa efeito entre indicadores de desempenho: uma análise a partir dos pressupostos do balanced scorecard. In: XVIII USP International Conference in Accounting. São Paulo: [s.n.]. 